EPJ Web of Conferences 21, 08006 (2012)

DOI: $10.1051 /$ epjconf/20122108006

(C) Owned by the authors, published by EDP Sciences, 2012

\title{
Brownian shape motion: Fission fragment mass distributions
}

\author{
Jørgen Randrup ${ }^{1, \text { a }}$, Peter Möller ${ }^{2}$, and Arnold J. Sierk ${ }^{2}$ \\ 1 Nuclear Science Division, Lawrence Berkeley National Laboratory, Berkeley, CA 94720, USA \\ 2 Theoretical Division, Los Alamos National Laboratory, Los Alamos, NM 87545, USA
}

\begin{abstract}
It was recently shown that remarkably accurate fission-fragment mass distributions can be obtained by treating the nuclear shape evolution as a Brownian walk on previously calculated five-dimensional potential-energy surfaces; the current status of this novel method is described here.
\end{abstract}

\section{Introduction}

As discussed already in the pioneering works by Meitner and Frisch [1] and Bohr and Wheeler [2,3] in 1939, nuclear fission can be viewed qualitatively as an evolution of the nuclear shape from that of a single compound nucleus to two receding fragments. The character of the shape dynamics is still not well established but, as a step away from a purely statistical approach toward a full dynamical treatment, it is interesting to explore scenarios in which the evolution is strongly dissipative $[4,5]$.

We therefore picture the nuclear fission process as an evolution of the nuclear shape from a relatively compact mononucleus to a dinuclear configuration. In the present study, the shape is composed of three smoothly joined quadratic surfaces $[6,7]$ which are described by a set of parameters, $\chi=\left\{\chi_{i}\right\}$, whose time development is the result of a complicated interplay between a variety of effects.

Most basic is the potential energy associated with a given shape, $U(\chi)$, for which a number of relatively mature models have been developed. We employ potential-energy surfaces that have been calculated with the macroscopic-microscopic method in which the potential energy is the sum of shapedependent macroscopic (liquid-drop type) terms and a microscopic correction that reflects the structure of the single-particle levels in the effective potential associated with the specified nuclear shape $[8,9]$. (Such potential energies have been calculated for 5,254 even-even nuclei with $170 \leq A \leq 330$ and tabulated on a five-dimensional Cartesian lattice containing over five milllion different shapes.) The potential energy provides the driving force, $\boldsymbol{F}^{\mathrm{pot}}(\chi)$, which has the components $F_{i}^{\mathrm{pot}}=-\partial U / \partial \chi_{i}$.

The driving force seeks to change the nuclear shape towards lower energies and the associated matter rearrangement gives rise to a collective kinetic energy, $\frac{1}{2} \dot{\boldsymbol{\chi}} \cdot \boldsymbol{M} \cdot \dot{\boldsymbol{\chi}}$. When the damping is strong, as we are assuming, the resulting motion is slow. In that scenario, the acceleration terms, $\left\{\sum_{j} M_{i j} \ddot{\chi}_{j}\right\}$, as well as terms of second order in the velocities $\left\{\dot{\chi}_{i}\right\}$ may be neglected. As a result, the inertial masses $\left\{M_{i j}\right\}$ play no role for the shape evolution and they shall henceforth be neglected.

Furthermore, the shape degrees of freedom are coupled dissipatively to the internal degrees of freedom in the nuclear many-body system. As a result, the shape evolution is both damped and diffusive and it must therefore be treated by transport methods that allow for the stochastic elements of the dynamics. The dynamics is then akin to Brownian motion: The Brownian particle is the (fivedimensional) nuclear shape, while the fluid into which it is immersed is represented by the internal (nucleonic) degrees of freedom to which it is coupled.

\footnotetext{
a e-mail: JRandrup@LBL . gov
}

This is an Open Access article distributed under the terms of the Creative Commons Attribution-Noncommercial License 3.0, which permits unrestricted use, distribution, and reproduction in any noncommercial medium, provided the original work is properly cited. 


\section{EPJ Web of Conferences}

\section{Brownian shape motion}

The average dissipative force provides a friction of the standard form, $\left\langle\boldsymbol{F}^{\mathrm{diss}}\right\rangle=\boldsymbol{F}^{\text {fric }}=-\boldsymbol{\gamma} \cdot \boldsymbol{F}$, and we assume that the random remainder, $\boldsymbol{F}^{\mathrm{ran}}(t)$, is Markovian, $\left\langle F_{i}^{\mathrm{ran}}(t) F_{j}^{\mathrm{ran}}\left(t^{\prime}\right)\right\rangle=2 T \gamma_{i j} \delta\left(t-t^{\prime}\right)$, where $T$ is the nuclear temperature at the particular shape $\chi$. The dissipation tensor $\gamma$ is positive definite and so is its inverse, the mobility tensor $\mu \equiv \gamma^{-1}$, which can be conveniently expressed in terms of its eigenvectors $\tilde{\chi}^{(n)}$

$$
\boldsymbol{\mu} \cdot \tilde{\chi}^{(n)}=\mu^{(n)} \tilde{\chi}^{(n)}, \quad \sum_{i} \tilde{\chi}_{i}^{(n)} \cdot \tilde{\chi}_{i}^{\left(n^{\prime}\right)}=\mu^{(n)} \delta_{n n^{\prime}}, \quad \mu_{i j}=\sum_{n} \tilde{\chi}_{i}^{(n)} \tilde{\chi}_{j}^{(n)} .
$$

The shape evolution $\chi(t)$ is governed by the Smoluchowski equation of motion which balances the driving force from the potential against the full dissipative force: $\boldsymbol{F}^{\mathrm{pot}}+\boldsymbol{F}^{\mathrm{fric}}+\boldsymbol{F}^{\mathrm{ran}} \doteq \mathbf{0}$. This condition immediately yields the instantaneous velocity,

$$
\dot{\chi}(t) \doteq \boldsymbol{\mu} \cdot\left[\boldsymbol{F}^{\mathrm{pot}}+\boldsymbol{F}^{\mathrm{ran}}(t)\right], \quad \delta \chi=\sum_{n} \tilde{\chi}^{(n)}\left[\Delta t \tilde{\chi}^{(n)} \cdot \boldsymbol{F}^{\mathrm{pot}}+\sqrt{2 T \Delta t} \xi_{n}\right] .
$$

The expression on the right-hand side shows how the change in $\chi$ accumulated during a brief time interval $\Delta t$ consists of an average displacement proportional to (but generally not aligned with ) the driving force and a stochastic term arising from the random force. On the basis of this expresison, it is straightforward to solve the Smoluchowski equation by direct numerical simulation. This type of evolution is exactly characteristic of Brownian motion.

In order to explore the dependence of the calculated mass distribution $P\left(A_{\mathrm{f}}\right)$ on the specific friction employed, we proceed as follows. We first invoke the simple wall formula for the one-body dissipation rate caused by the nuclear shape change [10] and calculate the associated dissipation tensor $\gamma(\chi)$,

$$
\dot{Q}=m \rho \bar{v} \int \dot{n}^{2} d \sigma=\sum_{i j} \dot{\chi}_{i} \gamma_{i j}(\chi) \dot{\chi}_{j}=\sum_{\mu \nu} \dot{q}_{\mu} \mathbf{g}_{\mu \nu}(\boldsymbol{q}) \dot{q}_{\nu}, \quad \gamma_{i j}(\chi)=\sum_{\mu \nu} \frac{\partial q_{\mu}}{\partial \chi_{i}} \mathbf{g}_{\mu \nu}(\boldsymbol{q}) \frac{\partial q_{v}}{\partial \chi_{j}}
$$

The coefficients $g_{\mu v}(\boldsymbol{q})$ can be directly evaluated in terms of the parameters $\left\{q_{v}\right\}$ characterizing 3QS shapes [6,7] employed for the calculation of $U(\chi)[8,9]$ and the needed elements $\gamma_{i j}$ can then be obtained by the appropriate transformation. Because we are not interested in the time evolution but merely in the path of the shape, the overall strength of $\gamma$ is immaterial, so we renormalize it at each lattice site so its eigenvalues are unity on average. Subsequently we generate an entire family of dissipation tensors, $\tilde{\gamma}(f)$, by modifying the eigenvalues, $\tilde{\gamma}_{n}^{(f)}(\chi) \equiv\left(\gamma_{n}(\chi)+f\right) /(1+f)$, so the friction becomes ever more isotropic as the parameter $f$ is increased from 0 to $\infty$.
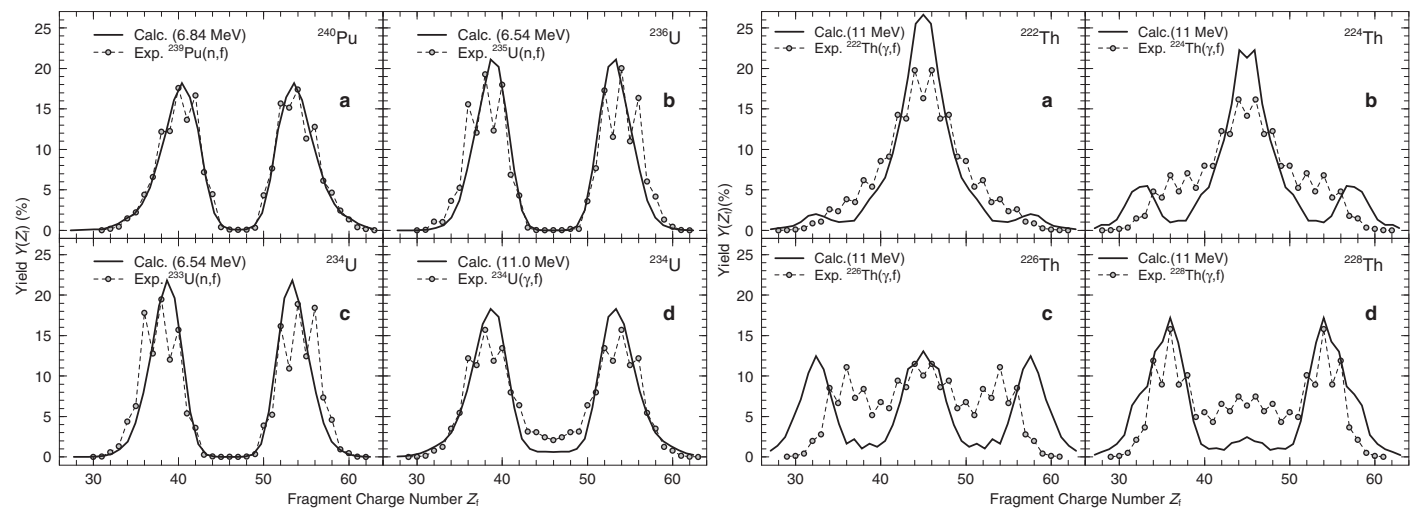

Fig. 1. Fragment charge distributions extracted from Metropolis walks on the respective 5D potential-energy surfaces [8,9], together with the corresponding experimental data [11,12]. (From Ref. [4].) 
$\mathrm{CNR} * 11$
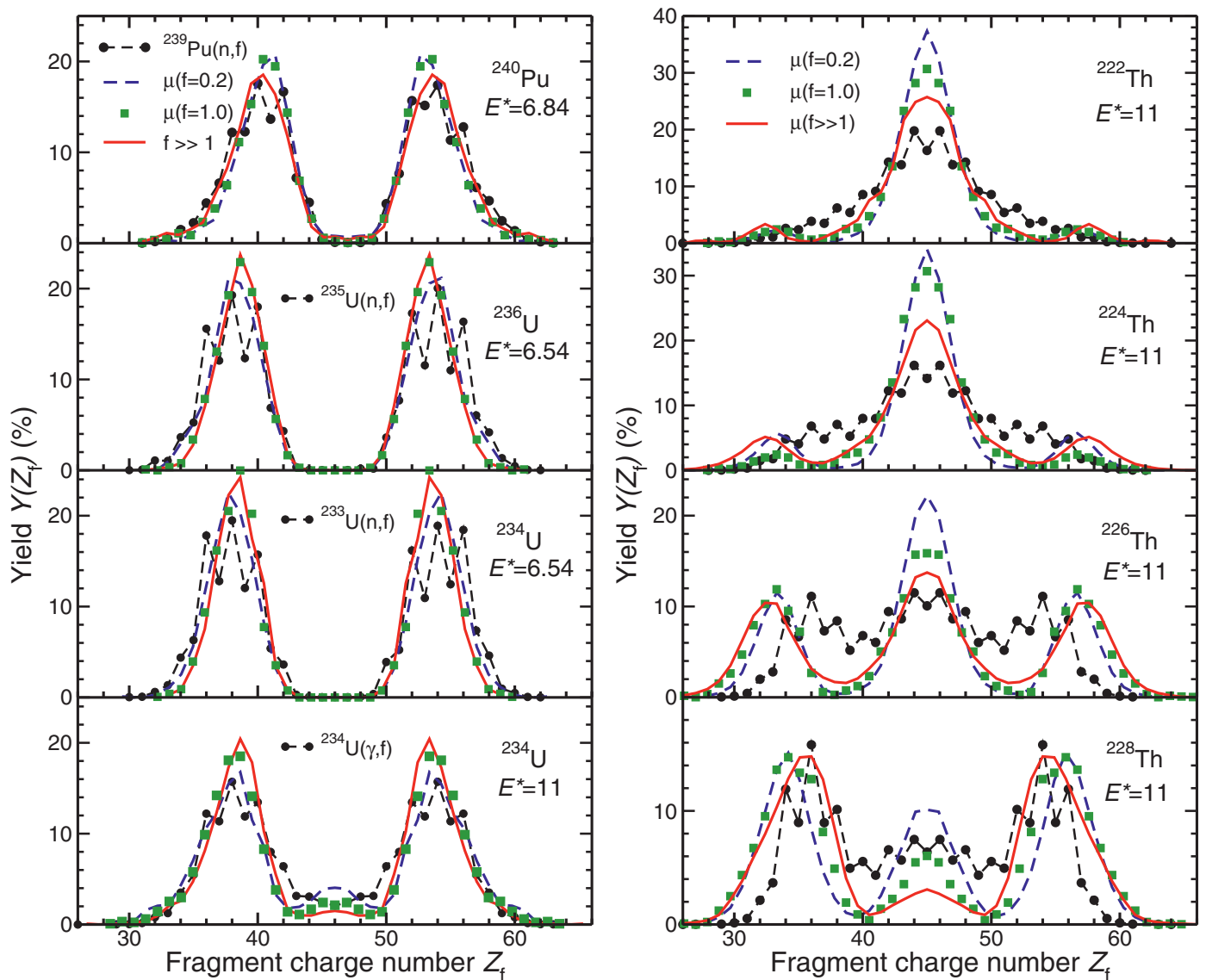

Fig. 2. The fragment charge distribution obtained by solving the Smoluchowski equation (2) for dissipation tensors having various anisotropies as specified by $f$, together with the experimental data [11,12]. (From Ref. [5].)

\section{Calculated mass distributions}

Once the dissipation tensor $\gamma$ has been specified, the Smoluchowski equation (2) is solved by numerical simulation starting from a suitable initial shape. In principle, the intial shape should be at (or near) the ground-state minimum, but the calculational effort can be reduced significantly by starting from a more distorted shape, without unduly influencing the results, and we normally start from the outer minimum, since all paths from the ground state to fission lead through that region. The calculation is then stopped when the neck radius has shrunk below a specified critical value $c_{0}$ and the mass asymmetry of the shape is assumed to give the final mass split. Several thousand such "events" suffice to obtain $P\left(A_{\mathrm{f}}\right)$ to within an acceptable tolerance; as shall be demonstrated, the resulting distribution is quite insensitive to the precise value of $c_{0}$.

In the original study [4], the fission-fragment mass distributions were obtained by performing a simple random walk by means of the Metropolis procedure, which provides an exact solution to the transport problem in the idealized (and unrealistic) case when the dissipation tensor is isotropic. Figure 1 shows the resulting charge distribution $P\left(Z_{\mathrm{f}}\right)=P\left(A_{\mathrm{f}}\right) A_{0} / Z_{0}$ for eight different cases, for which experimental data exist; for the thorium sequence, $P\left(Z_{\mathrm{f}}\right)$ changes qualitatively from symmetric to asymmetric. The overall agreement with the data is remarkably good, especially considering that no parameter was fitted. Even though the agreement for the thorium isotopes is not as perfect as for the plutonium and uranium cases, the deviations of the model results from the data are smaller than the differences between neighboring data sets, which in turn differ by just a single neutron orbital. 

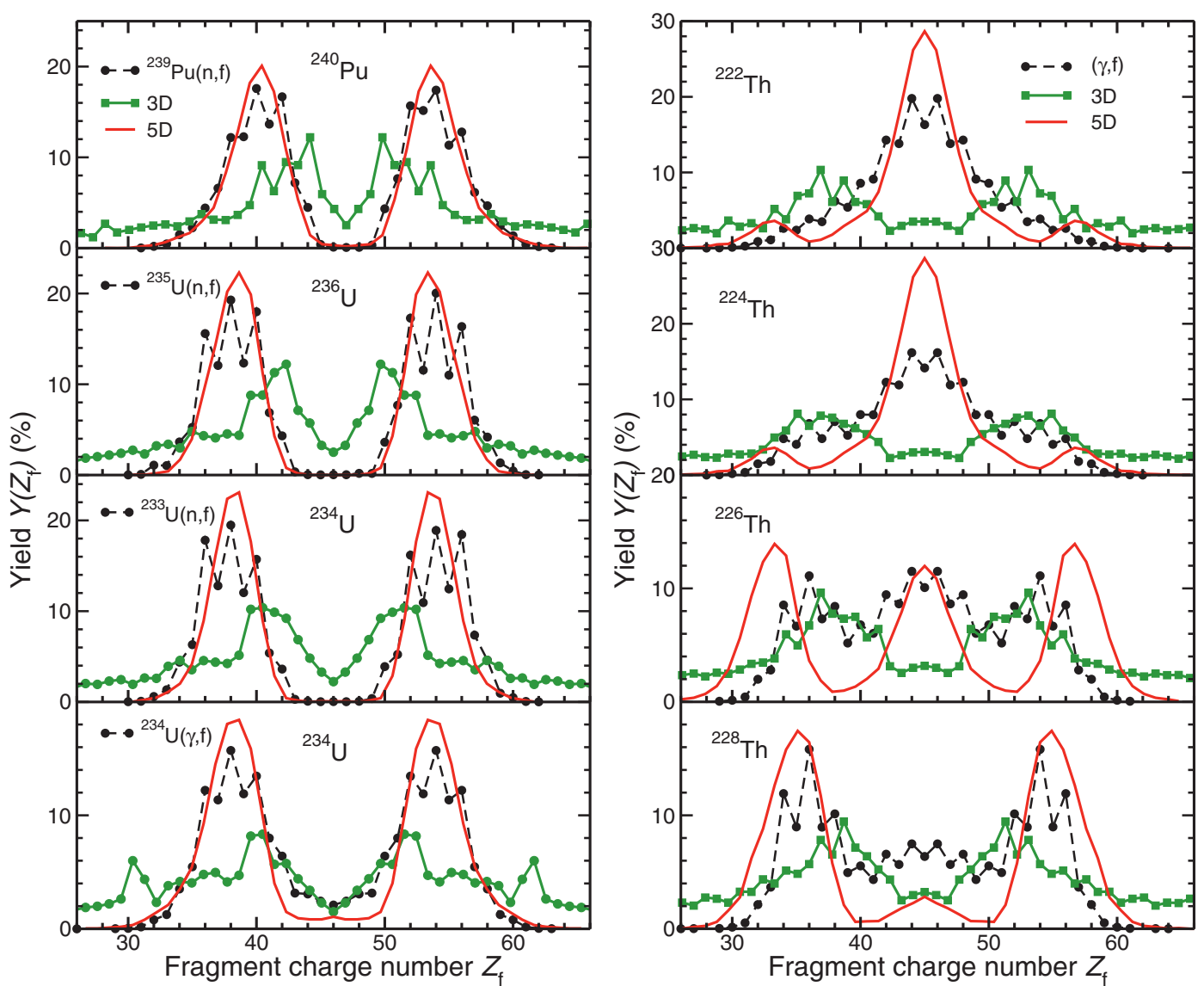

Fig. 3. Charge yields resulting from Metropolis walks on the $5 \mathrm{D}$ potential-energy surfaces, together with the corresponding results obtained with $3 \mathrm{D}$ surfaces generated by minimizing the $5 \mathrm{D}$ surfaces with respect to the individual fragment deformations, $\epsilon_{\mathrm{f} 1}$ and $\epsilon_{\mathrm{f} 2}$; the experimental data are from $[11,12]$. (From Ref. [5].)

The sensitivity of the calculated charge yields to the degree of structure in the mobility tensor is illustrated in Fig. 2. In addition to the experimental data, which are shown for reference, each plot shows the result of three different mobility scenarios: the idealized scenario (labeled $f \gg 1$ ) where the mobility tensor is isotropic, an intermediate scenario $(f=1)$ in which the dissipation tensor is the average of the one calculated with the wall formula (see Eq. (3)) and the corresponding directional average, and a more structured scenario $(f=0.2)$ in which the isotropic admixture is only $20 \%$.

\subsection{Shape family}

The three-quadratic-surface $(3 \mathrm{QS})$ shape family [6,7] can be described in terms of the overall elongation (corresponding to the quadrupole moment $Q_{2}$ ), the degree of constriction (which becomes the neck radius for binary shapes), the reflection asymmetry (determining the fragment mass $A_{\mathrm{f}}$ ), and the individual deformations of the two spheroidal parts $\left(\epsilon_{\mathrm{f} 1}\right.$ and $\left.\epsilon_{\mathrm{f} 2}\right)$ [8].

In order to illustrate the importance of employing a shape family that has a sufficient degree of flexibility, we construct three-dimensional potential-energy surfaces by minimizing the full fivedimensional 3QS surfaces with respect to $\epsilon_{\mathrm{f} 1}$ and $\epsilon_{\mathrm{f} 2}$ (corresponding to the lattice indices $K$ and $L$ ). Thus the shapes in the lower-dimensional space are characterized by only their overall elongation (represented by the lattice index $I$ ), their constriction (represented by the lattice index $J$ ), and the degree of reflection asymmetry (represented by the lattice index $M$ ). 


\section{CNR*11}

Figure 3 shows the resulting charge distributions, together with the experimental data and our standard results based on the full 5D 3QS shape family. We see that the although the 3D calculations occasionally reproduce the qualitative appearance of $P\left(Z_{\mathrm{f}}\right)$ reasonably well, the reproduction of the experimental data is generally far inferior to the results obtained with the 5D shape family. This suggests that it is of utmost importance to employ a shape family that has sufficient flexibility.

\subsection{Scission model}

As already recognized in the very earliest discussions of fission theory [2,3], induced fission involves as an intermediate stage the formation of a compound nucleus in which the excitation energy is distributed among the various degrees of freedom in a statistical manner. However, the compound equilibrium is established only among those relatively compact shapes that are situated inside of the fission barrier, and the ensemble experiences a continual leakage as some nuclei accidentally acquire a shape far enough beyond the saddle point that they then undergo an irreversible evolution towards scission. (Elementary phase-space estimates of the leakage rate form the basis for the familiar transition-state theory of fission.) Thus the resulting fission-fragment mass distribution bears no particular relationship to the distribution of the asymmetric modes of the compound-nuclear shapes. Furthermore, because of the non-equilibrium nature of the post-barrier evolution there is no reason to expect that the resulting
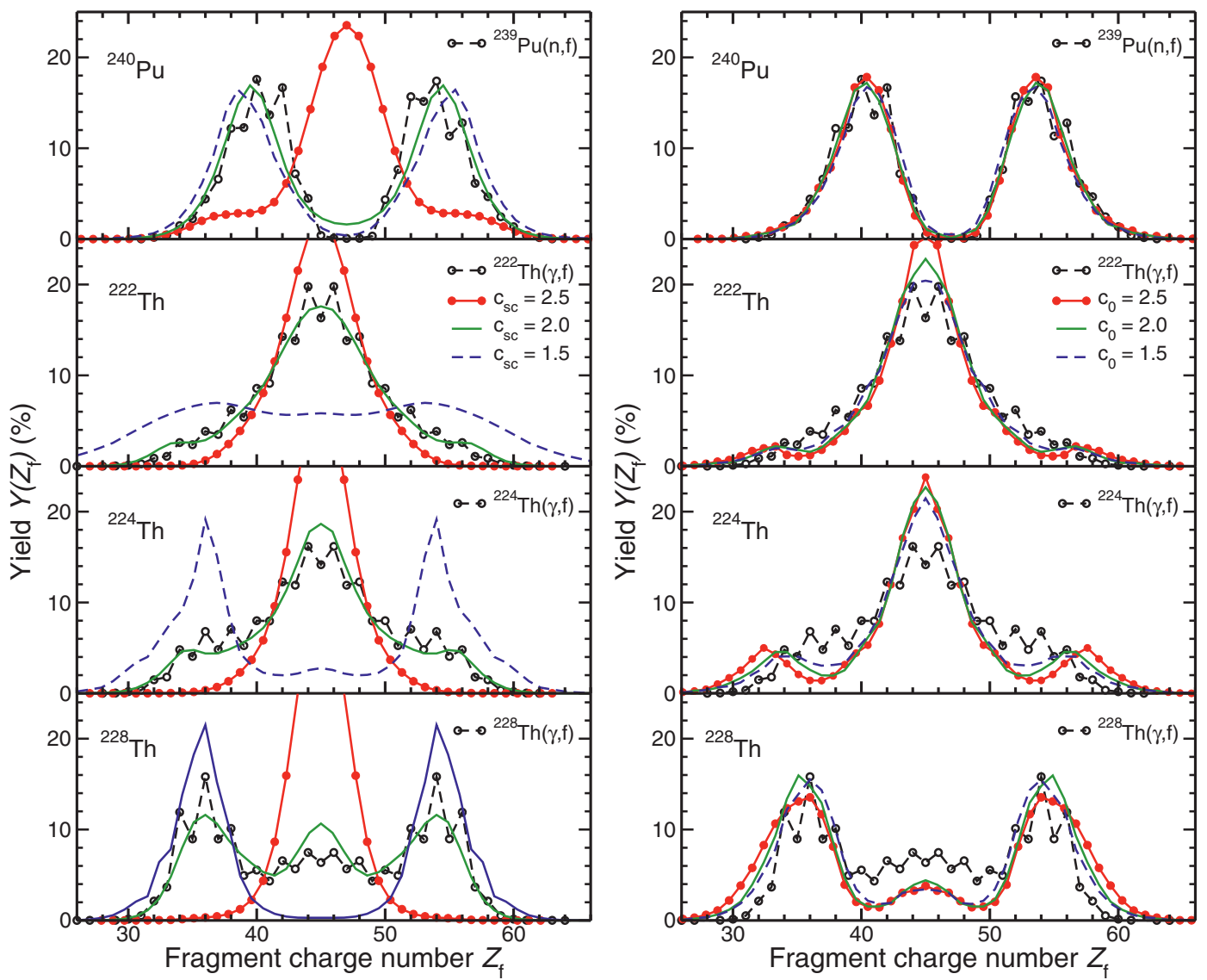

Fig. 4. Results of our scission model (see text) for ${ }^{240} \mathrm{Pu}$ and ${ }^{222,224,228} \mathrm{Th}$ for the scission neck radii $c_{\mathrm{sc}}=$ 2.5, 2.0, $1.5 \mathrm{fm}$ (left) and results of the Metropolis walk [4] for the same cases obtained with the same values of the critical neck radius $c_{0}(r i g h t)$; the experimental data are from [11,12]. (From Ref. [5].) 


\section{EPJ Web of Conferences}

mass distribution should have a statistical form. Nevertheless, a variety of statisical scission models have been studied. Indeed, models based on such considerations were among the earliest developed $[13,14]$ and, for each specific case, the details can often be adjusted to yield reasonable agreement with observed mass distributions. The importance of the pre-scission shape evolution can be illustrated by comparing the transport results with what a statistical population of the scission shapes would yield.

For this purpose, we define a four-dimensional scission hypersurface as consisting of those shapes $\{\tilde{\chi}\}$ for which the neck radius equals a specified value, $c(\tilde{\chi})=c_{\text {sc }}$, and we then assume that these scission configurations are populated in proportion to their statistical weight, $W(\tilde{\chi}) \sim \exp (-U(\tilde{\chi}) / T(\tilde{\chi}))$. As in the transport calculations, we use the shape-dependent temperature which ensures that the statistical weight drops rapidly to zero at the boundary of the energetically accessible region of shapes, namely those for which $U(\chi) \leq U_{\mathrm{gs}}+E^{*}$.

The results of such scission-model calculations are shown in Fig. 4 (left) for four representative cases, while Fig. 4 (right) shows the corresponding results obtained with the simple Metropolis walk [4]. For the scission model the character of the fragment mass distribution changes qualitatively as $c_{\mathrm{sc}}$ is decreased, whereas Metropolis walks yield mass distributions that are remarkably robust against the changes in the critical neck radius $c_{0}$. These results suggest that the mass split is not determined primarily by the energetics in the scission region but rather by the broader potential-energy landscape encountered by the shape during its pre-scission evolution.

\subsection{Specific illustrations}

The formal framework for the present treatment is considerably simpler than that used in a conventional Langevin-type dynamical treatment (for reviews, see Refs. [15, 16]). Accordingly, the information that can be extracted is correspondingly reduced. The key assumption of strong dissipation allows us to neglect the inertial masses and this in turn reduces the equation of motion from second to first order in time. The resulting transport equation pertains to the limit of creeping motion and thus provides only the path of the fissioning nucleus in the shape space, rather than its full temporal evolution. Furthermore, on the basis of our studies, it appears that the Metropolis walk, which is significantly faster than the Smoluchowski simulation (by 1-2 orders of magnitude), offers a very quick and easy means for obtaining practically useful fission-fragment mass distributions.
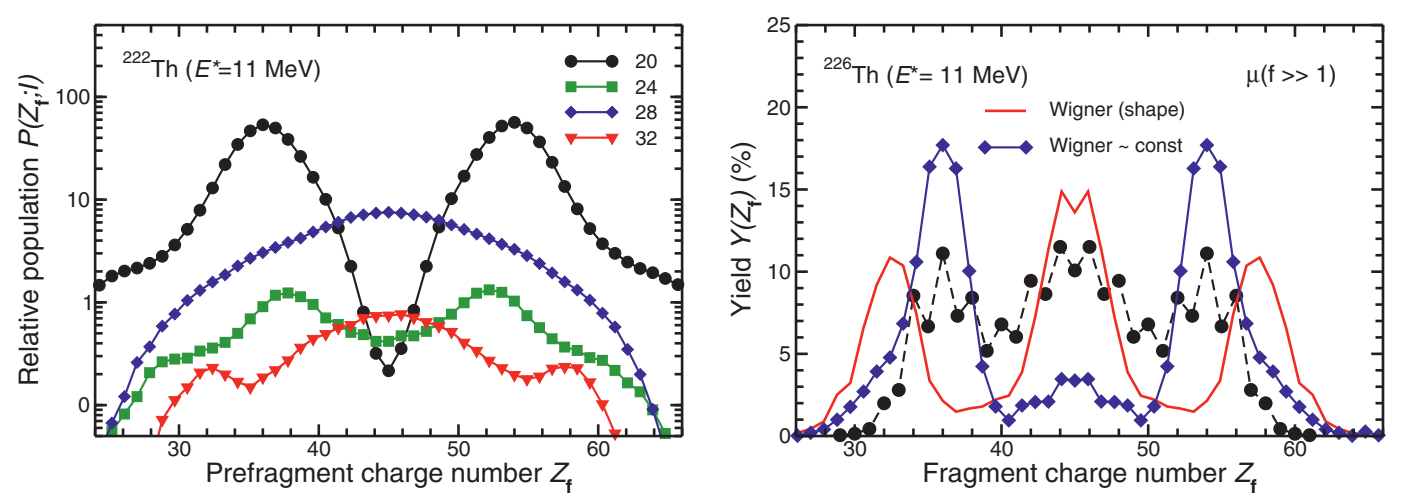

Fig. 5. Left: The evolution of the mass-asymmetry distribution from the region of the second saddle $(I=20)$ towards scission is illustrated by the charge distribution $P\left(Z_{\mathrm{f}} ; I\right)$ for increasing values of $I$ (the lattice index giving the quadrupole moment $Q_{2}$ of the nuclear shape), as obtained for ${ }^{222} \mathrm{Th}(11 \mathrm{MeV})$ from 10,000 Metropolis walks. Right: The sensitivity of the charge yields to the shape dependence of the Wigner term is illustrated for ${ }^{226} \mathrm{Th}$, using either the standard potential-energy surface [8,9], in which the Wigner term changes gradually as the shape evolves, or a modified energy surface obtained with a Wigner term that remains constant until scission occurs; calculated with an isotropic mobility tensor $(f \rightarrow \infty)$; the experimental data are also shown [12]. (From Ref. [5].) 


\section{$\mathrm{CNR} * 11$}

\subsubsection{Saddle shape}

Because our calculational method emulates the actual equilibration process it is possible to gain insight into the shape evolution during fission. A particularly instructive case is presented by ${ }^{22}$ Th for which the transport calculations yield a symmetric mass distribution, in agreement with the experimental data, even though they are based on a potential-energy surface whose fission saddle point corresponds to a nuclear shape that is reflection asymmetric. This result invalidates the commonly made assumption (see e.g. Refs. [17-20]) that the character of the mass distribution, whether symmetric or asymmetric, is determined by the character of the saddle shape. Rather, the structure of the potential-energy landscape in the entire region between the isomeric minimum and scission plays a role in determining the fragment mass distribution.

Detailed insight into this evolution can be obtained by considering the distribution of the charge asymmetry at specified values of the lattice index $I$ which is a measure of the overall quadrupole moment of the fissioning shape, $P\left(Z_{\mathrm{f}} ; I\right)$. This conditional distribution is shown in Fig. 5 (left) for increasing elongations. Because it is energetically favorable for the system to traverse the barrier with an asymmetry close to that of the saddle shape, $P\left(Z_{\mathrm{f}} ; I_{\text {saddle }}\right)$ is concentrated around that asymmetry. However, beyond the saddle the preferred asymmetry tends to become smaller $(I=24)$ and the distribution eventually becomes peaked at symmetry $(I=28)$. As the shape evolves further towards scission $(I=32), P\left(Z_{\mathrm{f}} ; I\right)$ developes a minor asymmetric component that presumably reflects the detailed (and possibly inaccurate) structure of the potential-energy surface in the scission region.

\subsubsection{Wigner term}

The generality of the treatment makes it possible to exploit any remaining differences between calculated results and experimental data to gain novel insight into aspects of the fission process that would not otherwise be readily accessible. As an example, we consider the shape dependence of the Wigner term in the macroscopic nuclear energy [21-26].

The macroscopic nuclear energy contains the so-called Wigner term proportional to $|N-Z| / A$. Its presence is clearly visible in the systematics of nuclear masses which exhibit a $V$ shape near isosymmetry, but its microscopic origin is still not well understood, so it is normally modeled as a phenomenological macroscopic term. In commonly employed models for nuclear masses [27,28] the Wigner term is usually introduced without a shape dependence. However, such an ansatz presents a significant (but often ignored) problem in fission where a single nucleus is transformed into two separate nuclei, each having its own Wigner term. (That each fragment nucleus must give a Wigner contribution similar to that of the original nucleus is evident from the phenomenological form of the term $[22,23]$.) Thus the Wigner term must double in magnitude during the evolution from a single compound system to two separated fragments, so, consequently, it must depend on the nuclear shape.

Since 1989 the calculations of fission barriers (see e.g. Refs. [9,22,26]) have employed a postulated shape dependence that relates the increase of the Wigner term to the decrease in the amount of communication between the two fragments due to the shrinking of the neck. The resulting Wigner term changes gradually as the nuclear shape evolves and affects the potential-energy landscape correspondingly (an illustrative figure was given in Ref. [26]). However, it may be argued that the change in the character of the fissioning system from mononuclear to dinuclear occurs more abruptly than implied by the currently prescribed shape dependence. To elucidate the importance of the shape dependence of the Wigner term, we have considered an alternate form in which the term is kept constant up to the scission configuration, i.e. until the neck radius has shrunk below the specified critical value $c_{0}$.

Figure 5 (right) illustrates how such a modification of the potential-energy surface affects the calculated charge distribution of ${ }^{226} \mathrm{Th}$, for which the impact is particularly noticeable. There are two significant differences between the results of the two sets of calculations. One is a change in the relative importance of symmetric and asymmetric fission, with the constant Wigner term leading to more asymmetric yield. The other is a shift in the location of the asymmetric yield peaks, from being several units on the outside of the observed values towards a better agreement with the data. Both effects depend significantly on the structure of the mobility tensor (see Ref. [5]) and could, in principle, be of help in discriminating between different forms of the dissipation. 
EPJ Web of Conferences

\section{Concluding remarks}

In conclusion, our studies suggest that the simple Metropolis walk [4] on the previously calculated potential-energy lattice $[8,9]$ indeed presents a useful calculational tool for obtaining the approximate form of fission-fragment mass distributions for a large range of nuclei. For more accurate results it is necessary to invoke also the dissipative features of the shape evolution as represented by the shapedependent mobility tensor. The shape evolution then resembles Brownian motion in an anisotropic (and non-uniform) medium. However, because the dissipation mechanism is not yet as well understood as the potential energy, we propose to make a series of calculations with mobility tensors that display different degrees of anisotropy and then use the ensuing spread in the results as an indication of the uncertainty of the predicted mass yield. The results obtained in this manner are often remarkably robust. Consequently, the method may be of practical use for calculating fission-fragment mass distributions for any of the thousands of nuclei for which the required 5D potential-energy surface is already available.

This work was supported by the Office of Nuclear Physics in the U.S. Department of Energy's Office of Science under Contract DE-AC02-05CH11231 (JR) and JUSTIPEN/UT grant DE-FG0206ER41407 (PM), and by the National Nuclear Security Administration of the U.S. Department of Energy at Los Alamos National Laboratory under Contract No. DE-AC52-06NA25396 (PM \& AJS).

\section{References}

1. L. Meitner and O.R. Frisch, Nature 143, 239 (1939).

2. N. Bohr, Nature 143, 330 (1939).

3. N. Bohr and J.A. Wheeler, Phys. Rev. 56, 426 (1939).

4. J. Randrup and P. Möller, Phys. Rev. Lett. 106, 132503 (2011).

5. J. Randrup, P. Möller, A.J. Sierk, Phys. Rev. C 84034613 (2011).

6. J.R. Nix, Report UCRL-17958, preprint of Ref. [7].

7. J.R. Nix, Nucl. Phys. A 130, 241 (1969).

8. P. Möller, D.G. Madland, A.J. Sierk, and A. Iwamoto, Nature 409, 785 (2000).

9. P. Möller, A.J. Sierk, T. Ichikawa, A. Iwamoto, R. Bengtsson, H. Uhrenholt, and S. Åberg, Phys. Rev. C 79, 064304 (2009).

10. J. Błocki, Y. Boneh, J.R. Nix, J. Randrup, M. Robel, A.J. Sierk, and W.J. Swiatecki, Ann. Phys. 113, 330 (1978).

11. M.B. Chadwick et al., Nucl. Data Sheets 107, 2931 (2006).

12. K.-H. Schmidt et al., Nucl. Phys. A 665, 221 (2000).

13. P. Fong, Phys. Rev. 102, 434 (1956).

14. B.D. Wilkins, E.P. Steinberg, and R.R. Chasman, Phys. Rev. C 14, 1832 (1976).

15. Y. Abe, S. Ayik, P.-G. Reinhard, and E. Suraud, Phys. Reports 275, 49 (1996).

16. P. Fröbrich and I.I. Gontchar, Phys. Reports 292, 131 (1998).

17. P. Möller, Nucl. Phys. A 192, 529 (1972).

18. M. Bolsterli, E.O. Fiset, J.R. Nix, and J.L. Norton, Phys. Rev. C 5, 1050 (1972).

19. J. Benlliure, A. Grewe, M. de Jong, K.-H. Schmidt, S. Zhdanov, Nucl. Phys. A 628 458, (1998).

20. K.-H. Schmidt, J. Benlliure, and A.R. Junghans, Nucl. Phys. A 693, 169 (2001).

21. W.D. Myers, Droplet model of atomic nuclei, (IFI/Plenum, New York, 1977).

22. P. Möller, J.R. Nix, and W.J. Swiatecki, Nucl. Phys. A 492, 349 (1989).

23. P. Möller and J.R. Nix, J. Phys. G 20, 1681 (1994).

24. W.D. Myers and W.J. Swiatecki, Nucl. Phys. A 601, 141 (1996).

25. W.D. Myers and W.J. Swiatecki, Nucl. Phys. A 612, 249 (1997).

26. P. Möller, A.J. Sierk, and A. Iwamoto, Phys. Rev. Lett. 92, 072501 (2004).

27. P. Möller, J.R. Nix, W.D. Myers, and W.J. Swiatecki, At. Data Nucl. Data Tables 59, 185 (1995).

28. S. Goriely, M. Samyn, P.-H. Heenen, J.M. Pearson, and F. Tondeur, Phys. Rev. 66, 024326 (2002). 